\title{
Analytical construction of a nonperturbative vacuum for the open bosonic string
}

\author{
V. Alan Kostelecký \\ Physics Department, Indiana University, Bloomington, Indiana 47405 \\ Robertus Potting \\ U.C.E.H., Campus de Gambelas, Universidade do Algarve, 8000 Faro, Portugal
}

(Received 31 August 2000; published 30 January 2001)

\begin{abstract}
Using analytical methods, a nonperturbative vacuum is constructed recursively in the field theory for the open bosonic string. Evidence suggests it corresponds to the Lorentz-invariant end point of tachyon condensation on a D25-brane. The corresponding string field is a twisted squeezed state.

DOI: 10.1103/PhysRevD.63.046007

PACS number(s): 11.25.Sq
\end{abstract}

\section{INTRODUCTION}

The physical behavior of strings can be investigated in a variety of ways. Much of the existing lore is based on perturbative studies of single strings in relativistic quantum mechanics. However, it is likely that a satisfactory understanding of the subject requires also mastering nonperturbative and collective string behaviors.

String field theory offers one framework within which to investigate nonperturbative many-body string phenomena. An essential feature of any field theory is the structure of its vacuum, and it is of particular interest to establish the existence of any nonperturbative vacua. A relatively simple string field theory describes the open bosonic string [1], and for this case the existence of at least one nonperturbative vacuum has been established [2-4]. The procedure involves a level-truncation scheme in which successive approximations to the full theory are made according to the mass level of the particle fields and the terms in the action. Analytical methods at low truncation orders and a combination of analytical and numerical methods at higher orders can determine the structure of the string field $\mathcal{N}$ for the nonperturbative vacuum in terms of expectation values of particle fields.

The nonperturbative vacuum $\mathcal{N}$ has been conjectured by Sen to be the end product of tachyon condensation on a D25-brane [5]. Strong support in favor of this conjecture exists from explicit calculations using the level-truncation scheme in the field theory for the open bosonic string [6-8], with these now having reached level $(10,20)$ in the notation of Ref. [4]. Analogous calculations for the superstring provide further support for the idea that the tachyon effective potential has a minimum where the D-brane tension is exactly cancelled [9-12].

In this work, we present an analytical approach to constructing the nonperturbative vacuum $\mathcal{N}$. We obtain a series representation for $\mathcal{N}$ and compare it to the numerical solution obtained via the truncation scheme. The methodology and results offer several interesting possibilities for future exploration. In the remainder of this introduction, we provide a brief summary of the steps involved in the construction of $\mathcal{N}$, and we offer some motivation for our procedure.

The steps involved in the construction of $\mathcal{N}$ are as follows. First, note that $\mathcal{N}$ is to be determined as a set of con- stant expectation values satisfying the string-field equations of motion:

$$
Q \mathcal{N}+g \alpha^{\prime} \mathcal{N} \star \mathcal{N}=0
$$

The requirement of spacetime independence of $\mathcal{N}$ makes it useful to transform the cubic vertex to a form in which the oscillator zero modes are converted into the momentum representation, so that the momentum may be set to vanish. Once this form of the cubic vertex is obtained, we search for a string field $\mathcal{S}$ obeying

$$
\mathcal{S} \star \mathcal{S}=c_{0} \mathcal{S},
$$

which serves as the basic object upon which the solution is to be constructed. We next perform a Bogoliubov transformation to a new oscillator basis for which $\mathcal{S}$ is the vacuum state. In this new basis, the string field $\mathcal{N}$ can be expanded in particle-field modes and the conditions determining their constant values can be extracted from the equations of motion (1). These conditions form a recursive set that is amenable to a formal solution for $\mathcal{N}$. Certain features of the solution can be compared to those already established via the level-truncation scheme, with good agreement.

The above procedure may appear somewhat convoluted at first sight, so we provide some heuristic physical and mathematical motivation before detailing the calculations in the following sections. On the physical side, one intuitively expects $\mathcal{N}$ to describe a situation in which the whole string field has condensed to the vacuum. It is therefore plausible that an infinite number of particle fields acquire vacuum values in the nonperturbative vacuum $\mathcal{N}$. In fact, it has been shown [13] that no nontrivial finite linear combination of expectation values for the particle fields can satisfy Eq. (1). Intuition suggests that the lighter modes should play a greater role in the development of the string-field condensate, so one might anticipate expectation values in $\mathcal{N}$ to drop with mass level. This is supported by evidence from numerical calculations with the level-truncation scheme. Furthermore, physical intuition about harmonic oscillators and the formation of a 
coherent condensate in the perturbative vacuum also suggests the natural form of the string field $\mathcal{N}$ is likely to be closely related to a generalized coherent or squeezed state [14]. These notions are echoed in our construction, since the solution we obtain for $\mathcal{S}$ in fact is a squeezed state and the nonperturbative vacuum $\mathcal{N}$ is developed by twisting $\mathcal{S}$ with oscillator factors controlled by the BRST operator $Q$.

On the mathematical side, we observe that the usual description in terms of perturbative oscillator modes and the level-truncation scheme itself use a basis for the Fock space in which $Q$ is essentially diagonal and the star product has a complicated realization. However, the structure of the string field equations can be viewed as analogous to a Riccati differential equation, involving a single derivative operator (basically, $L_{0} \Psi$ ), a linear term (the factor $-\Psi$ ), and a quadratic term (the product $\Psi \star \Psi$ ) [15]. In such equations the nonlinear term represents the greatest complication, and it is therefore natural to seek a representation in which the star product has a relatively simple structure. The ideal case would be to convert the quadratic term to linear form. This can be approximated by finding a string state $\mathcal{S}$ satisfying Eq. (2) and converting to a basis in which $\mathcal{S}$ is the basic (ground) state, so that the star product has a relatively simple realization. The expression for $Q \Psi$ becomes complicated in the new basis, so it might seem that little has been gained. However, the net effect of the manipulations is that the difficulty has been moved from the nonlinear part of the equation to the linear part, which provides just enough advantage to make possible the construction of a solution.

The remainder of the paper is organized as follows. In Sec. II, some preliminaries are discussed, including the conversion of the oscillator zero modes to the momentum representation and some properties of coherent and squeezed states. The reader uninterested in these details may wish to pass directly to Sec. III, in which the squeezed string field $\mathcal{S}$ is obtained. Section IV converts to the squeezed-oscillator basis. The construction of the nonperturbative vacuum $\mathcal{N}$ is presented in Sec. V, along with some of its properties. The results are discussed in Sec. VI. Finally, the Appendix contains a derivation of a useful identity. Throughout much of this work, the string coupling $g$ as defined in Ref. [2] and the string tension $\alpha^{\prime}$ are set to one, although they are explicitly displayed in certain formulas for clarity.

\section{PRELIMINARIES}

\section{A. Momentum representation}

The equations of motion of the string field can be expressed in terms of particle fields using a Fock-space representation [16-18]. We are interested in spacetimeindependent solutions of these equations, so it is useful to express the spacetime dependence of the vertex in the momentum representation rather than in the representation with oscillator zero modes. Following the approach of Ref. [16], we require the three-vertex $V_{3}$ involving the vertex functions $V_{m n}^{\prime r s}$ to satisfy

$$
\begin{aligned}
V_{3}= & \exp \left(-\frac{1}{2} \sum_{r, s} \sum_{m, n \neq 0} a_{m}^{r \dagger} V_{m n}^{\prime r s} a_{n}^{s \dagger}\right. \\
& \left.-\sum_{r, s} \sum_{n \neq 0} a_{0}^{r \dagger} V_{0 n}^{\prime r s} a_{n}^{s \dagger}-\frac{1}{2} \sum_{r} a_{0}^{r \dagger} V_{00}^{\prime r r} a_{0}^{r \dagger}\right)|0\rangle_{123} \\
\equiv & \int d p^{1} d p^{2} d p^{3} \exp \left(-\frac{1}{2} \sum_{r, s} \sum_{m, n \neq 0} a_{m}^{r \dagger} V_{m n}^{r s} a_{n}^{s \dagger}\right. \\
& \left.-\sum_{r, s} \sum_{n \neq 0} p^{r} V_{0 n}^{r s} a_{n}^{s \dagger}-\frac{1}{2} \sum_{r} p^{r} V_{00}^{r r} p^{r}\right)|0, p\rangle_{123},
\end{aligned}
$$

where the vertex functions $V_{m n}^{r s}$ determine $V_{3}$ in the momentum representation of interest. As usual, the $r, s$ superscripts are understood to take values modulo 3 .

Rewriting the right-hand side using

$$
|p\rangle=(2 \pi)^{-1 / 4} \exp \left[-\frac{1}{4} p^{2}+a_{0}^{\dagger} p-\frac{1}{2}\left(a_{0}^{\dagger}\right)^{2}\right]|0\rangle,
$$

we find the relations

$$
\begin{aligned}
& V_{m n}^{\prime r s}=V_{m n}^{r s}-2 \sum_{t} \frac{V_{m 0}^{r t} V_{0 n}^{t s}}{2 V_{00}^{t t}+1}, \quad m, n \neq 0, \\
& V_{0 n}^{\prime r s}=\frac{2 V_{0 n}^{r s}}{2 V_{00}^{r r}+1}, \quad n \neq 0, \\
& V_{00}^{\prime r r}=\frac{2 V_{00}^{r r}-1}{2 V_{00}^{r r}+1} .
\end{aligned}
$$

Next, we use these relations to derive some properties of the transformed vertex functions.

First, we note that the 'double square' of the vertex functions $V_{m n}^{\prime r s}$ generates the identity [16]:

$$
\sum_{k, t} V_{m k}^{\prime r t} V_{k n}^{\prime t s}=\delta^{r s} \delta_{m n}, \quad m, n=0,1, \ldots
$$

This leads to the following identities for the vertex functions in the momentum representation:

$$
\sum_{k \geqslant 1} \sum_{t} V_{m k}^{r t} V_{k n}^{t s}=\delta^{r s} \delta_{m n}, \quad m, n=1,2, \ldots
$$

$$
\begin{aligned}
& \sum_{k \geqslant 1} \sum_{t} V_{m k}^{r t} V_{k 0}^{t s}=V_{m 0}^{r s}, \quad n=1,2, \ldots, \\
& \sum_{k \geqslant 1} \sum_{t} V_{0 k}^{r t} V_{k 0}^{t s}=2 V_{00}^{r r} \delta^{r s} .
\end{aligned}
$$

In particular, we see from the first of these relations that the vertex function $V_{m n}^{r s}$, for which $m, n$ are restricted to nonzero level numbers, double squares to the identity in analogy with Eq. (6). 
Next, we establish some features of the structure of the transformed vertex functions $V_{m n}^{r s}$. Recall that the vertex functions $V_{m n}^{\prime r s}$ viewed as matrices can be expressed as [16]

$$
\begin{gathered}
V^{\prime r r}=\frac{1}{3}\left(C+U^{\prime}+\bar{U}^{\prime}\right), \\
V^{\prime r+1}=\frac{1}{3}\left(C+\alpha U^{\prime}+\alpha^{*} \bar{U}^{\prime}\right), \\
V^{\prime r+2}=\frac{1}{3}\left(C+\alpha^{*} U^{\prime}+\alpha \bar{U}^{\prime}\right),
\end{gathered}
$$

where $\alpha=\exp (2 \pi i / 3), C_{m n} \equiv(-1)^{m} \delta_{m n}$, and the matrices $U^{\prime}$ and $\bar{U}^{\prime} \equiv C U^{\prime} C$ satisfy

$$
\left(U^{\prime}\right)^{2}=\left(\bar{U}^{\prime}\right)^{2}=1, \quad\left(U^{\prime}\right)^{\dagger}=U^{\prime} .
$$

Some algebra shows that these relations imply

$$
\begin{aligned}
V^{r r} & =\frac{1}{3}(C+U+\bar{U}), \\
V^{r+1} & =\frac{1}{3}\left(C+\alpha U+\alpha^{*} \bar{U}\right), \\
V^{r+2} & =\frac{1}{3}\left(C+\alpha^{*} U+\alpha \bar{U}\right),
\end{aligned}
$$

where

$$
U_{m n}=U_{m n}^{\prime}+\left(V_{00}^{r r}+\frac{1}{2}\right) U_{m 0}^{\prime} U_{0 n}^{\prime}, \quad m, n=1,2, \ldots,
$$

and $\bar{U}=C U C$. It also follows that

$$
U^{2}=\bar{U}^{2}=1, \quad U^{\dagger}=U,
$$

as before. These equations imply that the matrix $V^{r r} C$ $=C V^{r r}=\frac{1}{3}(1+U C+C U)$ commutes with all the vertexfunction matrices $V^{r s}$, while $C V^{r s}=V^{s r} C$. These identities are used below.

The above arguments establish that the vertex functions $V_{m n}^{r s}$ restricted to $n, m \geqslant 1$ have the same formal structure as the original vertex functions $V_{m n}^{\prime r s}$. This means that searches for spacetime-independent solutions to the equations of motion involve the same formal vertex structure as spacetimedependent ones. We return to this point in Sec. VI.

In the remainder of this paper except where otherwise stated, the indices $m, n$ range over $1,2, \ldots$, so the results are independent of momentum. Thus, for example, the bosonic part of the three-vertex $V_{3}$ takes the form

$$
\left|V_{3}^{b}\right\rangle=\exp \left(-\frac{1}{2} \sum_{r, s=1}^{3} a_{m}^{r \dagger} V_{m n}^{r s} a_{n}^{s \dagger}\right)|0\rangle_{123} .
$$

\section{B. Identities for coherent and squeezed states}

This section presents some identities required in subsequent sections of the paper, where frequent use is made of generalized squeezed states. First, recall a basic identity for coherent states in one oscillator dimension,

$$
\left\langle 0\left|\exp (\lambda a) \exp \left(\mu a^{\dagger}\right)\right| 0\right\rangle=\exp (\lambda \mu),
$$

and the corresponding identity for squeezed states,

$$
\left\langle 0\left|\exp \left(\frac{1}{2} a S a\right) \exp \left(\frac{1}{2} a^{\dagger} V a^{\dagger}\right)\right| 0\right\rangle=(1-S V)^{-1 / 2} .
$$

Combining Eqs. (14) and (15) to an identity for displaced squeezed states in one oscillator dimension yields

$$
\begin{aligned}
\langle 0| \exp & \left(\lambda a+\frac{1}{2} a S a\right) \exp \left(\mu a^{\dagger}+\frac{1}{2} a^{\dagger} V a^{\dagger}\right)|0\rangle \\
= & (1-S V)^{-1 / 2} \exp \left[\lambda(1-V S)^{-1} \mu\right. \\
& \left.+\frac{1}{2} \lambda(1-V S)^{-1} V \lambda+\frac{1}{2} \mu(1-S V)^{-1} S \mu\right] .
\end{aligned}
$$

The analogous identity for $\langle 0| \exp \left(\lambda_{n} a_{n}+\frac{1}{2} a_{m} S_{m n} a_{n}\right)$ $\times \exp \left(\mu_{n} a_{n}^{\dagger}+\frac{1}{2} a_{m}^{\dagger} V_{m n} a_{n}^{\dagger}\right)|0\rangle$ in the multidimensional case is expected to have the form

$$
\begin{aligned}
\left\langle 0\left|\exp \left(\lambda \cdot a+\frac{1}{2} a \cdot S \cdot a\right) \exp \left(\mu \cdot a^{\dagger}+\frac{1}{2} a^{\dagger} \cdot V \cdot a^{\dagger}\right)\right| 0\right\rangle \\
=\operatorname{Det}(1-S \cdot V)^{-1 / 2} \exp \left[\lambda \cdot(1-V \cdot S)^{-1} \cdot \mu\right. \\
\left.\quad+\frac{1}{2} \lambda \cdot(1-V \cdot S)^{-1} \cdot V \cdot \lambda+\frac{1}{2} \mu \cdot(1-S \cdot V)^{-1} \cdot S \cdot \mu\right],
\end{aligned}
$$

where the dot indicates contraction of indices. This can be shown explicitly in two dimensions, for example, by making repeated use of Eq. (16).

In the fermionic case, with an anticommuting oscillator algebra [19]

$$
\left\{b_{m}, c_{n}^{\dagger}\right\}=\left\{c_{m}, b_{n}^{\dagger}\right\}=\delta_{m n}, \quad m, n=1,2, \ldots,
$$

and all other anticommutators zero, the equivalent of Eq. (17) can be found as

$$
\begin{gathered}
\left\langle 0\left|\exp (-b \cdot \widetilde{S} \cdot c) \exp \left(b^{\dagger} \cdot \mu^{b}+\mu^{c} \cdot c^{\dagger}+b^{\dagger} \cdot \widetilde{V} \cdot c^{\dagger}\right)\right| 0\right\rangle \\
=\operatorname{Det}(1-\widetilde{S} \cdot \widetilde{V}) \exp \left[\mu^{c} \cdot(1-\widetilde{S} \cdot \widetilde{V})^{-1} \cdot \widetilde{S} \cdot \mu^{b}\right] .
\end{gathered}
$$

For simplicity, we have taken the analogue of the parameter $\lambda$ to vanish here.

Consider next the star product $\mathcal{S}_{1} \star \mathcal{S}_{2}$ of two squeezed states, $\mathcal{S}_{1} \equiv \exp \left(\frac{1}{2} a^{1 \dagger} \cdot S^{11} \cdot a^{1 \dagger}\right)|0\rangle_{1}$ with squeeze matrix $S^{11}$ and $\mathcal{S}_{2} \equiv \exp \left(\frac{1}{2} a^{2 \dagger} \cdot S^{22} \cdot a^{2 \dagger}\right)|0\rangle_{2}$ with squeeze matrix $S^{22}$. The result can be written as

$$
\begin{aligned}
\left|\mathcal{S}_{1} \star \mathcal{S}_{2}\right\rangle_{3} \equiv & { }_{12}\langle 0| \exp \left(\frac{1}{2} a^{1} \cdot S^{11} \cdot a^{1}+\frac{1}{2} a^{2} \cdot S^{22} \cdot a^{2}\right) \\
& \times \exp \left(\mu^{1} \cdot a^{1 \dagger}+\mu^{2} \cdot a^{2 \dagger}\right. \\
& \left.+\frac{1}{2} \sum_{r, s=1}^{2} a^{r \dagger} \cdot V^{r s} \cdot a^{s \dagger}\right)|0\rangle_{123},
\end{aligned}
$$

where $\mu^{1} \equiv a^{3 \dagger} V^{31}, \mu^{2} \equiv a^{3 \dagger} V^{32}$. Evaluating first the expectation value of the 1-oscillators using (17) on the levelnumber indices $m, n$ and then evaluating the expectation value of the 2-oscillators produces a somewhat cumbersome expression. However, it simplifies in the special case for which the matrices $S^{11} C$ and $S^{22} C$ commute with $V^{r s}$. In the 
next section, it is shown that this case is the relevant one for the construction of solutions of the string field equations. With the simplification, the result of the calculation is

$$
\begin{aligned}
\left|\mathcal{S}_{1} \star \mathcal{S}_{2}\right\rangle_{3}= & {\left[\operatorname{Det}_{m n}\left(\operatorname{Det}_{r s}(1-\Sigma V)\right)\right]^{-1 / 2} } \\
& \times \exp \left[\sum_{r, s=1}^{2} \mu^{2}\left[(1-\Sigma V)^{-1} \Sigma\right]^{r s} \mu^{s}\right]|0\rangle_{3},
\end{aligned}
$$

where we have introduced a matrix $\Sigma^{r s}$ in the twodimensional string indices given by

$$
\Sigma^{r s}=\left(\begin{array}{cc}
S^{11} & 0 \\
0 & S^{22}
\end{array}\right)
$$

In Eq. (21), note the double determinant that occurs in terms of the string indices $r, s$ and the level-number indices $m, n$.

In the ghost sector, the three-vertex takes the form [20]

$$
\left|V_{3}^{g h}\right\rangle=\exp \left[\sum_{r, s=1}^{3} b_{m}^{r \dagger}\left(E \widetilde{V}^{r s} E^{-1}\right)_{m n} c_{n}^{s \dagger}\right]|+\rangle_{123},
$$

where the matrix $E$ is given by $E_{m n}=\delta_{m n} / \sqrt{m}$. In this equation, the matrices $\widetilde{V}^{r s}$ have the form

$$
\begin{gathered}
\widetilde{V}^{r r}=\frac{1}{3}(C+\widetilde{U}+\overline{\tilde{U}}), \\
\widetilde{V}^{r+1}=\frac{1}{3}\left(C+\alpha \widetilde{U}+\alpha^{*} \overline{\tilde{U}}\right), \\
\widetilde{V}^{r+2}=\frac{1}{3}\left(C+\alpha^{*} \widetilde{U}+\alpha \overline{\tilde{U}}\right),
\end{gathered}
$$

with $\overline{\tilde{U}}=C \tilde{U} C, \widetilde{U}^{2}=\overline{\tilde{U}}^{2}=1$, and $\widetilde{U}^{\dagger}=\widetilde{U}$, in complete analogy with the bosonic sector. Introducing matrices $\widetilde{S}^{11}$ and $\widetilde{S}^{22}$ such that $\widetilde{S}^{11} C$ and $\widetilde{S}^{22} C$ commute with $\widetilde{V}^{r s}$, the star product of two squeezed states is given in this case by

$$
\begin{aligned}
& { }_{12}\langle-| \exp \left(b^{1} \cdot E \widetilde{S}^{11} E^{-1} \cdot c^{1}+b^{2} \cdot E \widetilde{S}^{22} E^{-1} \cdot c^{2}\right) \\
& \times \exp \left(\mu^{c 1} E^{-1} \cdot c^{1 \dagger}+b^{1 \dagger} \cdot E \mu^{b 1}+\mu^{c 2} E^{-1} \cdot c^{2 \dagger}\right. \\
& \left.\quad+b^{2 \dagger} \cdot E \mu^{b 2}+\sum_{r, s=1}^{2} b^{r \dagger} \cdot E \widetilde{V}^{r s} E^{-1} \cdot c^{s \dagger}\right)|+\rangle_{123} \\
& =\left[\operatorname{Det}_{m n}\left(\operatorname{Det}_{r s}(1-\widetilde{\Sigma} \widetilde{V})\right)\right] \\
& \quad \times \exp \left[\sum_{r, s=1}^{2} \mu^{c r}\left[(1-\widetilde{\Sigma} \widetilde{V})^{-1} \Sigma\right]^{r s} \mu^{b s}\right]|+\rangle_{3} .
\end{aligned}
$$

In this equation, we have set

$$
\widetilde{\Sigma}^{r s}=\left(\begin{array}{cc}
\widetilde{S}^{11} & 0 \\
0 & \widetilde{S}^{22}
\end{array}\right),
$$

in analogy with the bosonic sector.

\section{THE SQUEEZED STRING FIELD $\mathcal{S}$}

In this section, we obtain squeezed-state solutions of Eq. (2). The nonperturbative vacuum $\mathcal{N}$ is constructed in Sec. V using these solutions.

We have shown in Eqs. (21) and (25) that the star product of two squeezed states is again a squeezed state. To solve Eq. (2), we require invariance of the matrix defining the width of the squeezed state. In the bosonic sector, this leads to the condition

$$
\mathrm{CSC}=\left(V^{12}, V^{21}\right)(1-\Sigma V)^{-1} \Sigma\left(\begin{array}{l}
V^{21} \\
V^{12}
\end{array}\right)+V^{11},
$$

where we have chosen

$$
\Sigma^{r s}=\left(\begin{array}{ll}
S & 0 \\
0 & S
\end{array}\right)
$$

In Eq. (27), the matrices $V^{12}=V^{31}$ and $V^{21}=V^{32}$ and their conjugates arise from the coefficients $\mu_{r} \equiv a^{3 \dagger} V^{3 r}$ in (21). The term $(1-\Sigma V)^{-1} \Sigma$ is to be interpreted as carrying both level-number indices $m, n=1,2, \ldots$ and string indices $r, s$ $=1,2$. The $C$ matrices multiplying $S$ on the left-hand side emerge from the application of the two vertex on the original state.

The challenge is to solve Eq. (27) for $S$, thereby determining the width of the squeezed state satisfying Eq. (2). It is convenient first to develop some machinery controlling the commutation of the various matrices on the right-hand side of Eq. (27).

Using the decomposition of the vertex functions $V^{r s}$ in terms of the matrices $C$ and $U$, the following identities can be shown to hold:

$$
V^{r s} C=C V^{s r}
$$

$$
\begin{aligned}
& \left(V^{12}, V^{21}\right)\left(\begin{array}{ll}
V^{11} & V^{12} \\
V^{21} & V^{11}
\end{array}\right)=-V^{11}\left(V^{12}, V^{21}\right) \\
& \left(V^{21}, V^{12}\right)\left(\begin{array}{ll}
V^{11} & V^{12} \\
V^{21} & V^{11}
\end{array}\right)=2 V^{11}\left(V^{12}, V^{21}\right)+C\left(V^{21}, V^{12}\right)
\end{aligned}
$$

To simplify the structure of the calculation, we introduce a two-dimensional space

$$
\left(\begin{array}{l}
A \\
B
\end{array}\right) \equiv A\left(V^{12}, V^{21}\right)+B\left(V^{21}, V^{12}\right)
$$

where $A$ and $B$ are scalars assumed to commute with all other expressions. In terms of this formalism, the relations (29) are

$$
\begin{aligned}
& \left(\begin{array}{l}
A \\
B
\end{array}\right) C=C\left(\begin{array}{l}
B \\
A
\end{array}\right), \\
& \left(\begin{array}{l}
A \\
B
\end{array}\right) V=\left(\begin{array}{cc}
-V^{11} & 2 V^{11} \\
0 & C
\end{array}\right)\left(\begin{array}{l}
A \\
B
\end{array}\right) .
\end{aligned}
$$


These equations can be used to determine the result of commuting the $C$ and $V$ matrices in Eq. (27) to the left.

As a further simplification, we make the ansatz

$$
S=C T,
$$

where $T$ is taken to commute with $V^{r s}$. This implies only the product $C V$ need be moved to the left in Eq. (27). Combining Eqs. (31) and (32) gives

$$
\left(\begin{array}{l}
A \\
B
\end{array}\right) C V=\left(\begin{array}{cc}
2 V^{11} C & -V^{11} C \\
1 & 0
\end{array}\right)\left(\begin{array}{l}
A \\
B
\end{array}\right) \equiv \Lambda\left(\begin{array}{l}
A \\
B
\end{array}\right) .
$$

Commuting $(1-\Sigma V)^{-1}=(1-C T V)^{-1}$ to the left yields $(1-T \Lambda)^{-1}$, which can be explicitly evaluated by diagonalizing the matrix $\Lambda$. In particular, in the case of interest $A$ $=1, B=0$ one finds

$$
\left(\begin{array}{l}
1 \\
0
\end{array}\right)(1-C T V)^{-1}=\left[1+V^{11} C T(T-2)\right]^{-1}\left(\begin{array}{l}
1 \\
T
\end{array}\right) .
$$

We thus obtain the result

$$
\begin{aligned}
\left(V^{12}, V^{21}\right)(1-C T V)^{-1} C T\left(\begin{array}{l}
V^{21} \\
V^{12}
\end{array}\right) \\
=\left[1+V^{11} C T(T-2)\right]^{-1} C T\left[\left(V^{21}, V^{12}\right)\right. \\
\left.\quad+T\left(V^{12}, V^{21}\right)\right]\left(\begin{array}{c}
V^{21} \\
V^{12}
\end{array}\right) \\
=\left[1+V^{11} C T(T-2)\right]^{-1} C T\left[2\left(V^{11}\right)^{2}-2 V^{11} C\right. \\
\left.\quad+T\left(1-\left(V^{11}\right)^{2}\right)\right] .
\end{aligned}
$$

Here, we used the identities

$$
\begin{aligned}
& \left(V^{21}\right)^{2}=\left(V^{12}\right)^{2}=\left(V^{11}\right)^{2}-V^{11} C, \\
& V^{12} V^{21}+V^{21} V^{12}=1-\left(V^{11}\right)^{2},
\end{aligned}
$$

which can be proved via the explicit expressions in terms of $U$ and $C$. Note that the result in Eq. (36) involves only $T, C$ and $V^{11}$.

We can use the result (36) in Eq. (27) to obtain an equation for $T$. Some rearrangement of terms yields

$$
(T-1)\left[T^{2}-\left(1+X^{-1}\right) T+1\right]=0,
$$

where $X \equiv C V^{11}$. This generates three solutions for $T$ :

$$
T=1, T_{ \pm}=\frac{1}{2 X}(1+X \pm \sqrt{(1+3 X)(1-X)}),
$$

validating the ansatz (33). The $T=1$ solution gives $S=C$, and the corresponding string field is the identity functional restricted to the bosonic sector. This solution is expected, and its appearance serves as a nice check on the formalism. However, it is physically irrelevant because it is unnormalizable.

The other two solutions are real for eigenvalues $x$ of $X$ satisfying $-\frac{1}{3} \leqslant x \leqslant 1$. The solution $T_{-}$behaves as $X$ for small $x$, while $T_{+}=1 / T_{-}$behaves as $X^{-1}$. A numerical check on the eigenvalues of $X=C V^{11}$ shows they are smaller than one and converge toward zero, with the three largest being $0.21,0.09,0.03$. Since the presence of any eigenvalue of $T$ with absolute value greater than or equal to one leads to an unnormalizable state, we consider only the solution $T_{-}$.

In the ghost sector, a similar construction can be performed. Starting with a general squeezed state defined by the matrix $\widetilde{S}$ and requiring that its width be invariant under the star product generates the condition

$$
-C \widetilde{S} C=\left(\widetilde{V}^{12}, \widetilde{V}^{21}\right)(1-\widetilde{\Sigma} \widetilde{V})^{-1} \widetilde{\Sigma}\left(\begin{array}{c}
\widetilde{V}^{21} \\
\widetilde{V}^{12}
\end{array}\right)+\widetilde{V}^{11},
$$

where

$$
\widetilde{\Sigma}^{r s}=\left(\begin{array}{cc}
\widetilde{S} & 0 \\
0 & \widetilde{S}
\end{array}\right)
$$

Note the minus sign on the left-hand side of Eq. (40), appearing because conjugation using the two-vertex introduces a relative minus sign for the $b$ and $c$ oscillators.

The solution of Eq. (40) is completely analogous to the solution of Eq. (27). This is because the matrices $\widetilde{V}^{r s}$ are defined through matrices $C$ and $\widetilde{U}$ as in the bosonic case, and so all identities leading to Eqs. (38) and (39) have ghostsector equivalents.

Writing $\widetilde{S}=C \widetilde{T}$ and $\widetilde{X}=C \widetilde{V}^{11}$, one obtains

$$
(\widetilde{T}+1)\left[\widetilde{T}^{2}+\left(\widetilde{X}^{-1}-3\right) \widetilde{T}+1\right]=0 .
$$

This has solutions

$$
\widetilde{T}=-1, \quad \widetilde{T}_{ \pm}=\frac{1}{2 \widetilde{X}}(3 \widetilde{X}-1 \pm \sqrt{(1-\widetilde{X})(1-5 \widetilde{X})}) .
$$

The expected piece of the unnormalizable identity functional is again found as a solution, corresponding to $\widetilde{T}=-1$.

The two solutions $\widetilde{T}_{ \pm}$are real for all $\widetilde{X}$ except for eigenvalues $\widetilde{x}$ in the range $\frac{1}{5}<\widetilde{x}<1$. For small $\widetilde{x}, \widetilde{T}_{+} \approx-\widetilde{X}$ is small while $\widetilde{T}_{-} \approx \widetilde{X}^{-1}$ is large. Since the eigenvalues of $\widetilde{V}^{11}$ converge toward zero, only $\widetilde{T}_{+}$can lead to a normalizable solution to the ghost sector of Eq. (2). One might wonder whether some of the eigenvalues $\tilde{x}$ of $\tilde{X}$ lie in the interval $\frac{1}{5}<\widetilde{x}<1$, which would lead to complex eigenvalues for $\widetilde{T}_{+}$. However, all the $\tilde{x}$ are negative, with the largest three eigenvalues being $-0.66,-0.25$, and -0.08 .

Combining the above results provides the squeezed state obeying Eq. (2) as

$$
\begin{aligned}
|\mathcal{S}\rangle= & \operatorname{Det}\left(1-S^{2}\right)^{1 / 4} \operatorname{Det}\left(1-\widetilde{S}^{2}\right)^{-1 / 2} \\
& \times \exp \left(\frac{1}{2} a^{\dagger} S a^{\dagger}\right) \exp \left(b^{\dagger} E \widetilde{S} E^{-1} c^{\dagger}\right)|+\rangle .
\end{aligned}
$$


By construction, it is an element of the star subalgebra of string fields. A family of wedge states also lying in this subalgebra was recently introduced in Ref. [22]. It would be interesting to determine explicitly the relationship of $\mathcal{S}$ to these wedge states.

\section{CONVERSION TO THE SQUEEZED BASIS}

Since the star product is idempotent on the string field $\mathcal{S}$, one can expect the form of the vertex function to be substantially simplified in a new oscillator basis chosen such that the squeezed state $|\mathcal{S}\rangle$ plays the role of the ground state. As before, we begin our considerations in the bosonic sector and subsequently extend them to the ghosts.

Observing that $\left(a-S a^{\dagger}\right) \exp \left(\frac{1}{2} a^{\dagger} S a^{\dagger}\right)|0\rangle=0$, we are motivated to define a new annihilation operator $s$ by the Bogoliubov transformation

$$
s=w\left(a-S a^{\dagger}\right), \quad a=w\left(s+S s^{\dagger}\right),
$$

where

$$
w=\left(1-S^{2}\right)^{-1 / 2} .
$$

The operator $s$ annihilates $|\mathcal{S}\rangle$, so the latter can be identified as the vacuum $|0\rangle_{s}$ in the $s$-oscillator basis:

$$
\begin{aligned}
& |0\rangle_{s}=\operatorname{Det}(w)^{-1 / 2} \exp \left(\frac{1}{2} a^{\dagger} S a^{\dagger}\right)|0\rangle_{a}, \\
& |0\rangle_{a}=\operatorname{Det}(w)^{-1 / 2} \exp \left(-\frac{1}{2} s^{\dagger} S s^{\dagger}\right)|0\rangle_{s} .
\end{aligned}
$$

Applying this transformation to the bosonic sector of the three vertex, we find

$$
\begin{aligned}
\left|V_{3}\right\rangle & =\exp \left(\frac{1}{2} a^{r \dagger} V^{r s} a^{s \dagger}\right)|0\rangle_{a, 123} \\
& \propto \exp \left(\frac{1}{2} a^{r \dagger}\left(V^{r s}-S \delta^{r s}\right) a^{s \dagger}\right)|0\rangle_{s, 123} \\
& \propto \exp \left[\left(s^{\dagger}+s S\right)^{r}\{w(V-S) w\}^{r s}\left(s^{\dagger}+S s\right)^{s}\right]|0\rangle_{s, 123} .
\end{aligned}
$$

In the last line of this equation and in what follows, it is understood that the symbol $S$ is to be interpreted as $S_{m n} \delta^{r s}$. In the Appendix, the useful identity

$$
\begin{aligned}
\exp \left(a^{\dagger} A a^{\dagger}+a^{\dagger} C a+a B a\right) \\
=\operatorname{Det}\left[(1-C) e^{C}\right]^{-1 / 2} \exp \left[a^{\dagger}(1-C)^{-1} A a^{\dagger}\right] \\
\quad \times \exp \left[-a^{\dagger} \ln (1-C) a\right] \exp \left[a B(1-C)^{-1} a\right]
\end{aligned}
$$

is shown to hold for multidimensional oscillators, where the matrices $A, B$, and $C$ satisfy

$$
A^{T}=A, \quad B^{T}=B, \quad A C^{T}=C A, \quad B C=C^{T} B, \quad C^{2}=4 A B .
$$

The identity (49) can be used to rewrite Eq. (48), since the appropriate identifications of $A, B, C$ satisfy the conditions (50). Noting that exponentials involving the annihilation operator $s$ act as the identity on the vacuum, we find

$$
\left|V_{3}\right\rangle \propto \exp \left(\frac{1}{2} s^{r \dagger} \hat{V}^{r s} s^{s \dagger}\right)|0\rangle_{s, 123},
$$

where

$$
\hat{V}=(1-V S)^{-1}(V-S)
$$

is the transformed three-vertex function.

Next, we investigate the properties of $\hat{V}$. Using the explicit form (10), $V^{r s}$ can be diagonalized in the $r, s$ indices by a matrix $\mathcal{O}$ satisfying $\mathcal{O}^{-1}=\mathcal{O}^{\dagger}$ :

$$
V=\mathcal{O}^{-1} V_{D} \mathcal{O}
$$

with

$$
V_{D}=\left(\begin{array}{ccc}
C & 0 & 0 \\
0 & U & 0 \\
0 & 0 & \bar{U}
\end{array}\right), \quad \mathcal{O}=\frac{1}{\sqrt{3}}\left(\begin{array}{ccc}
1 & 1 & 1 \\
\alpha^{*} & \alpha & 1 \\
\alpha & \alpha^{*} & 1
\end{array}\right) .
$$

For the transformed three-vertex $\hat{V}$, we find

$$
\hat{V}=\mathcal{O}^{-1}\left(1-V_{D} S\right)^{-1}\left(V_{D}-S\right) \mathcal{O}
$$

with the $r r$ elements of the diagonalized form given by

$$
\begin{aligned}
& \left(\left(1-V_{D} S\right)^{-1}\left(V_{D}-S\right)\right)^{11}=(1-C S)^{-1}(C-S)=C, \\
& \left(\left(1-V_{D} S\right)^{-1}\left(V_{D}-S\right)\right)^{22}=(1-U S)^{-1}(U-S), \\
& \left(\left(1-V_{D} S\right)^{-1}\left(V_{D}-S\right)\right)^{33}=(1-\bar{U} S)^{-1}(\bar{U}-S) .
\end{aligned}
$$

The diagonal elements of the transformed vertex function $\hat{V}^{r s}$ are all equal and given as one-third of the sum of its eigenvalues in Eqs. (56), in analogy with $V^{r s}$. Multiplying any one diagonal element $\hat{V}^{r r}$ from the left and from the right with $(1-U S)$ gives $V^{11}\left(1+S^{2}\right)-V^{11} C S-S$, which vanishes for the solution $S=C T_{-}$in Eq. (39). This is to be expected, since the $s$ vacuum can satisfy $|0\rangle_{s} \star|0\rangle_{s}=|0\rangle_{s}$ only if indeed the diagonal elements of $\hat{V}$ vanish.

The off-diagonal elements of $\hat{V}$ are also of interest. Explicitly, we find

$$
\hat{V}=\left(\begin{array}{ccc}
0 & \hat{V}^{12} & \hat{V}^{21} \\
\hat{V}^{21} & 0 & \hat{V}^{12} \\
\hat{V}^{12} & \hat{V}^{21} & 0
\end{array}\right),
$$

where

$$
\begin{aligned}
\hat{V}^{12} & =\frac{1}{3}\left(C+\alpha \Lambda+\alpha^{*} \bar{\Lambda}\right), \\
\hat{V}^{21} & =\frac{1}{3}\left(C+\alpha^{*} \Lambda+\alpha \bar{\Lambda}\right), \\
\Lambda & =(1-U S)^{-1}(U-S), \\
\bar{\Lambda} & =(1-\bar{U} S)^{-1}(\bar{U}-S)=C \Lambda C .
\end{aligned}
$$

It can be shown that 


$$
C+\Lambda+\bar{\Lambda}=0, \quad \Lambda^{2}=\bar{\Lambda}^{2}=1
$$

Using these results, some straightforward algebra yields

$$
\left(\hat{V}^{12} \hat{V}^{21}\right)^{2}=\hat{V}^{12} \hat{V}^{21}, \quad\left(\hat{V}^{21} \hat{V}^{12}\right)^{2}=\hat{V}^{21} \hat{V}^{12},
$$

demonstrating that

$$
P_{1} \equiv \hat{V}^{12} \hat{V}^{21}, \quad P_{2} \equiv \hat{V}^{21} \hat{V}^{12}
$$

are projection operators. It also follows that $P_{1}+P_{2}=1$, which implies $P_{1}$ and $P_{2}$ are conjugate. Other useful identities are

$$
\begin{gathered}
P_{1}=\hat{V}^{12} C=C \hat{V}^{21}, \quad P_{2}=\hat{V}^{21} C=C \hat{V}^{12}, \\
\hat{V}^{12}=\hat{V}^{12} P_{2}=P_{1} \hat{V}^{12}, \quad \hat{V}^{21}=\hat{V}^{21} P_{1}=P_{2} \hat{V}^{21}, \\
\left(\hat{V}^{12}\right)^{2}=\left(\hat{V}^{21}\right)^{2}=0 .
\end{gathered}
$$

We interpret the physical meaning of Eqs. (60) through (62) in terms of the behavior of the left half (" 1 ") and the right half ("2") of the string. Thus, $\hat{V}^{12}$ maps the right half onto the left half and annihilates the left half, while $\hat{V}^{21}$ does the converse. The operators $P_{1}$ and $P_{2}$ project onto the left and right halves of the string, respectively. This interpretation is consistent with the identity $\hat{V}^{12}+\hat{V}^{21}=C$, since the operator $C$ indeed interchanges the left and right halves of the string. The existence of such an interpretation is to be expected because the action of the three vertex is to map the left half of string $r$ onto the right half of string $r+1$ modulo 3 . The Bogoliubov transformation to the $s$ oscillator basis apparently generates a structure reminiscent of the comma representation proposed in Ref. [23]. It would be interesting to obtain explicitly the relation between the two formulations.

Turning next to the ghost sector, we define new fermionic ghost oscillators $t$ and $u$ through the Bogoliubov transformations

$$
\begin{gathered}
t=\left(b E-b^{\dagger} E \widetilde{S}\right) \widetilde{w} E^{-1}, \quad u=E \widetilde{w}\left(E^{-1} c-\widetilde{S} E^{-1} c^{\dagger}\right), \\
b=\left(t E+t^{\dagger} E S\right) \widetilde{w} E^{-1}, \quad c=E \widetilde{w}\left(E^{-1} u+S E^{-1} u^{\dagger}\right), \\
\widetilde{w}=\left(1-\widetilde{S}^{2}\right)^{-1 / 2} .
\end{gathered}
$$

The oscillators $t, u$ satisfy the same algebra as the $b, c$ oscillators. Also, $t, u$ annihilate the state $\exp \left(b^{\dagger} \widetilde{S} c^{\dagger}\right)|+\rangle_{b, c}$, so we identify the vacuum $|+\rangle_{t, u}$ as

$$
\begin{aligned}
& |+\rangle_{t, u}=\operatorname{Det}(\widetilde{w}) \exp \left(b^{\dagger} E \widetilde{S} E^{-1} c^{\dagger}\right)|+\rangle_{b, c}, \\
& |+\rangle_{b, c}=\operatorname{Det}(\widetilde{w}) \exp \left(-t^{\dagger} E \widetilde{S} E^{-1} u^{\dagger}\right)|+\rangle_{t, u} .
\end{aligned}
$$

The ghost three-vertex can be expressed in terms of the new oscillators:

$$
\begin{aligned}
\left|V_{3}^{g h}\right\rangle \propto & \exp \left[\left(t^{\dagger} E-t E \widetilde{S}\right)^{r}[\widetilde{w}(\widetilde{V}-\widetilde{S}) \widetilde{w}]^{r s}\right. \\
& \left.\times\left(E^{-1} u^{\dagger}+S E^{-1} u\right)^{s}\right]|+\rangle_{t, u, 123} .
\end{aligned}
$$

We find that the ghost version of the identity (49) for multidimensional oscillators is

$$
\begin{aligned}
& \exp \left(b^{\dagger} A c^{\dagger}+b^{\dagger} C c+c^{\dagger} C b+b B c\right) \\
&=\operatorname{Det}\left[(1-C) e^{c}\right] \exp \left[b^{\dagger}(1-C)^{-1} A c^{\dagger}\right] \\
& \times \exp \left[-b^{\dagger} \ln (1-C) c-c^{\dagger} \ln (1-C) b\right] \\
& \times \exp \left[b B(1-C)^{-1} c\right],
\end{aligned}
$$

where the matrices $A, B$, and $C$ satisfy

$A^{T}=A, \quad B^{T}=B, \quad A C^{T}=C A, \quad B C=C^{T} B, \quad C^{2}=-A B$.

Using the identity (66) in Eq. (65) yields

$$
\left|V_{3}^{g h}\right\rangle \propto \exp \left[t^{\dagger} E(1-\widetilde{V} \widetilde{S})^{-1}(\widetilde{V}-\widetilde{S}) E^{-1} u^{\dagger}\right]|+\rangle_{t, u, 123} .
$$

In analogy with the bosonic sector, it can be shown that the transformed ghost vertex

$$
\hat{\tilde{V}}=(1-\widetilde{V} \widetilde{S})^{-1}(\widetilde{V}-\widetilde{S})
$$

has zero diagonal $r r$ elements. Its off-diagonal elements can be used to define projection operators

$$
\widetilde{P}_{1}=\hat{V}^{12} \hat{V}^{21}, \quad \widetilde{P}_{2}=\hat{V}^{21} \hat{V}^{12} .
$$

The interpretation in terms of left and right halves of the string also holds here.

\section{THE NONPERTURBATIVE VACUUM $\mathcal{N}$}

\section{A. Construction}

We next consider the equations of motion for the string field and construct a solution for the nonperturbative vacuum $\mathcal{N}$. To simplify the notation in expansions of string fields, we use Greek indices to indicate a composite index containing both level-number indices and spacetime Lorentz indices. In the squeezed-state basis, a general string field $|f\rangle$ can then be expanded as

$$
\begin{aligned}
|f\rangle= & \sum_{m, n=0}^{\infty} f_{m, n}^{\lambda_{1} \ldots \lambda_{m} \mu_{1} \nu_{1} \cdots \mu_{n} \nu_{n}} \\
& \times s_{\lambda_{1}}^{\dagger} \cdots s_{\lambda_{m}}^{\dagger} t_{\mu_{1}}^{\dagger} u_{\nu_{1}}^{\dagger} \cdots t_{\mu_{n}}^{\dagger} u_{\nu_{n}}^{\dagger}|1-\rangle_{s, t, u} .
\end{aligned}
$$

Here, $f_{m, n}$ is a tensor that is totally symmetric in $\lambda_{1} \cdots \lambda_{m}$ and totally antisymmetric in $\mu_{1} \cdots \mu_{n}$ and in $\nu_{1} \cdots \nu_{n}$. The indices $m, n$ here label the number of composite bosonic and ghost indices rather than oscillator numbers, the latter being subsumed into Greek indices as explained above.

We are interested in solving the string equation of motion $Q \Psi+g \alpha^{\prime} \Psi \star \Psi=0$, which for $g=\alpha^{\prime}=1$ reduces in the Feynman-Siegel gauge [21] to 


$$
c_{0}\left(L_{0}-1\right) \Psi+\Psi \star \Psi=0 .
$$

Restricting attention first to the bosonic sector, the action of $L_{0}$ in the $s$-oscillator basis is found to be

$$
\begin{aligned}
L_{0}|f\rangle= & \sum_{m=0}^{\infty}\left[\left(s^{\dagger} w S E^{-2} w s^{\dagger}\right) f_{m 0}^{\lambda_{1} \cdots \lambda_{m}} s_{\lambda_{1}}^{\dagger} \cdots s_{\lambda_{m}}^{\dagger}\right. \\
& +m(m-1)\left(w S E^{-2} w\right)_{\lambda_{m-1} \lambda_{m}} f_{m 0}^{\lambda_{1} \cdots \lambda_{m}} s_{\lambda_{1}}^{\dagger} \cdots s_{\lambda_{m-2}}^{\dagger} \\
& +m\left(s^{\dagger} w\left(E^{-2}+S E^{-2} S\right) w\right)_{\lambda_{m}} f_{m 0}^{\lambda_{1} \cdots \lambda_{m}} s_{\lambda_{1}}^{\dagger} \cdots s_{\lambda_{m-1}}^{\dagger} \\
& \left.+\Delta f_{m 0}^{\lambda_{1} \cdots \lambda_{m}} s_{\lambda_{1}}^{\dagger} \cdots s_{\lambda_{m}}^{\dagger}\right]|0\rangle_{s} .
\end{aligned}
$$

Here, $\Delta$ is the energy of the squeezed $s, t, u$ vacuum relative to the usual $a, b, c$ vacuum, as measured by the action of $L_{0}$ and arising from normal ordering in the squeezed vacuum. It is given by

$$
\begin{aligned}
\Delta & =26 \Delta^{s}+\Delta^{t, u}, \\
\Delta^{s} & =\operatorname{Tr}\left(w S E^{-2} S w\right)=\operatorname{Tr}\left[S^{2}\left(1-S^{2}\right)^{-1} E^{-2}\right], \\
\Delta^{t, u} & =-2 \operatorname{Tr}\left[\widetilde{S}^{2}\left(1-\widetilde{S}^{2}\right)^{-1} E^{-2}\right] .
\end{aligned}
$$

The star product of two states $|f\rangle$ and $|g\rangle$ restricted to the bosonic sector is

$$
\begin{aligned}
|f \star g\rangle= & \sum_{m, n} \sum_{k=0}^{\min (m, n)}\left[\left(\hat{V}^{21}\right)_{\lambda_{1} \mu_{1}} \cdots\left(\hat{V}^{21}\right)_{\lambda_{k} \mu_{k}}\right] \\
& \times\left[\left(P_{1}\right)_{\lambda_{k+1}} \lambda_{k+1}^{\prime} \cdots\left(P_{1}\right)_{\lambda_{m}} \lambda_{m}^{\prime}\right] \\
& \times\left[\left(P_{2}\right)_{\mu_{k+1}} \mu_{k+1}^{\prime} \cdots\left(P_{2}\right)_{\mu_{n}}^{\mu_{n}^{\prime}}\right] \\
& \times f_{m 0}^{\lambda_{1} \cdots \lambda_{m}} g_{n 0}^{\mu_{1} \cdots \mu_{n}} s_{\lambda_{k+1}^{\dagger}}^{\dagger} \cdots s_{\lambda_{m}^{\prime}}^{\dagger} s_{\mu_{k+1}^{\prime}}^{\dagger} \cdots s_{\mu_{n}^{\prime}}^{\dagger}|0\rangle_{s} .
\end{aligned}
$$

Define the excitation number $m$ of a term in the field expansion as the associated number of $s^{\dagger}$ factors acting on the $s$ vacuum. Then, Eq. (73) reveals that $L_{0}$ maps excitation number $m$ to excitation numbers $m-2, m$, and $m+2$, while Eq. (75) shows that the star operator connects excitation numbers $m$ and $n$ to a combination of excitation numbers $m+n, m+n-2, \ldots,|m-n|$. The latter is reminiscent of the combination of two angular momenta. This is no accident, as we demonstrate next.

In the $s$-oscillator basis, the existence of the projection operators $P_{1}$ and $P_{2}$ separates the Greek indices into degrees of freedom pertaining to left and right halves of the string. This split enables the realization of an SU(2) symmetry associated with excitation number. Consider the operators

$$
\begin{aligned}
& J_{0}=\frac{1}{2}\left(s^{\dagger} P_{1} s-s^{\dagger} P_{2} s\right), \\
& J_{+}=s^{\dagger} \hat{V}^{12} s, \quad J_{-}=s^{\dagger} \hat{V}^{21} s .
\end{aligned}
$$

These form the generators of the excitation su(2) algebra. Note that the $J_{ \pm}$connect left and right oscillators. The total spin $J=s^{\dagger} s / 2$ characterizes the representations. This means that states of excitation number $n$ constitute a representation of the excitation $\mathrm{SU}(2)$ symmetry with $\operatorname{spin} J=n / 2$.

In general, the star product between states of excitation numbers $m$ and $n$ contains only $\min (m, n)+1$ nonzero states of the $m n$ possibilities, consisting of states of excitation $|m-n|$ through $m+n$. As an example, Eq. (75) shows the star product of two $J=1 / 2$ representations $|f\rangle_{s} \equiv f_{10}^{\lambda} s_{\lambda}^{\dagger}|0\rangle_{s}$ and $|g\rangle_{s} \equiv g_{10}^{\lambda} s_{\lambda}^{\dagger}|0\rangle_{s}$ is

$$
|f \star g\rangle=f_{10}^{\lambda} g_{10}^{\mu}\left[\left(\hat{V}^{21}\right)_{\lambda \mu}+\left(P_{1} s^{\dagger}\right)_{\lambda}\left(P_{2} s^{\dagger}\right)_{\mu}\right]|0\rangle_{s} .
$$

Two nonzero states remain, one of excitation 0 and one of excitation 2 .

The above discussion suggests it may be useful to distinguish even and odd excitation numbers. In particular, the action of both $L_{0}$ and the star operator are closed in the subsector of even excitation number. In the remainder of this section, we restrict attention to this subsector.

Despite the simplifications offered by the squeezed basis, obtaining an explicit exact solution to Eq. (72) remains a somewhat formidable task given the complexity of Eqs. (73) and (75). The approach we adopt here relies on the observation that the matrix $S$ appears in the definition of $L_{0}$. Following the discussion in Sec. III, it can be shown that the eigenvalues of $S$ are small. The largest eigenvalues are approximately $-0.21,0.09$, and -0.03 , displaying an alternating series converging to zero. It is therefore a reasonable strategy to solve Eq. (72) perturbatively in $S$.

We expand the desired string field $\mathcal{N}$ as

$$
|\mathcal{N}\rangle=\left|\mathcal{N}^{(0)}\right\rangle+\left|\mathcal{N}^{(1)}\right\rangle+\left|\mathcal{N}^{(2)}\right\rangle+\cdots
$$

where the superscript indicates the order in $S$. Substitution in Eq. (72) at order $S^{0}$ gives

$$
\begin{aligned}
& \sum_{m=0}^{\infty}\left(m s^{\dagger} E^{-2}-s^{\dagger}\right)_{\lambda_{1}} s_{\lambda_{2}}^{\dagger} \cdots s_{\lambda_{m}}^{\dagger} \mathcal{N}_{m 0}^{\lambda_{1} \cdots \lambda_{m}(0)}|0\rangle_{s} \\
& +\left|\mathcal{N}^{(0)}\right\rangle \star\left|\mathcal{N}^{(0)}\right\rangle=0 .
\end{aligned}
$$

Note that the energy of the squeezed vacuum $\Delta \sim \mathcal{O}\left(S^{2}\right)$ is irrelevant at this order. One solution is obtained by setting to zero all terms with $m \neq 0$, yielding

$$
\left|\mathcal{N}^{(0)}\right\rangle+\left|\mathcal{N}^{(0)}\right\rangle \star\left|\mathcal{N}^{(0)}\right\rangle=0
$$

We can use the analysis in Sec. IV to set

$$
\left|\mathcal{N}^{(0)}\right\rangle=|\mathcal{S}\rangle \equiv|0\rangle_{s},
$$

so that

$$
\mathcal{N}_{00}^{(0)}=1, \quad \mathcal{N}_{20}^{\lambda_{1} \lambda_{2}(0)}=\mathcal{N}_{40}^{\lambda_{1} \cdots \lambda_{4}(0)}=\cdots=0 .
$$

There may also be other possible choices producing different solutions to the string equations of motion. We return to this issue in the next section.

At order $S^{1}$, one obtains 


$$
\begin{aligned}
& {\left[\left(s^{\dagger} S E^{-2} s^{\dagger}\right) \mathcal{N}_{00}^{(0)}\right.} \\
& \left.\quad+\sum_{m}\left(m s^{\dagger} E^{-2}-s^{\dagger}\right)_{\lambda_{1}} s_{\lambda_{2}}^{\dagger} \cdots s_{\lambda_{m}}^{\dagger} \mathcal{N}_{m 0}^{\lambda_{1} \cdots \lambda_{m}(1)}\right]|0\rangle_{s} \\
& \quad+\left|\mathcal{N}^{(1)}\right\rangle \star\left|\mathcal{N}^{(0)}\right\rangle+\left|\mathcal{N}^{(0)}\right\rangle \star\left|\mathcal{N}^{(1)}\right\rangle=0,
\end{aligned}
$$

where the star product is understood to be expanded in components at the appropriate order, according to Eq. (75). Note that the star product of $\left|\mathcal{N}^{(0)}\right\rangle=|0\rangle_{s}$ with a term in $\mathcal{N}^{(1)}$ of excitation number $m$ yields a result with the same excitation number $m$. The presence of the first (inhomogeneous) term in Eq. (83) means that the only nonvanishing contribution at order $S$ involves excitation number 2 . In particular, $\mathcal{N}_{00}^{(1)}$ $=0$.

The explicit solution of Eq. (83) for $\left|\mathcal{N}^{(1)}\right\rangle$ yields a somewhat cumbersome expression, involving left and right projection operators. However, the solution at order $S$ simplifies greatly in the limit of high mass level, because the single term involving $m E^{-2}$ dominates all terms other than the first. In this limit, we find

$$
\mathcal{N}_{20}^{\lambda_{1} \lambda_{2}(1)} \approx-\frac{1}{2} S^{\lambda_{1} \lambda_{2}}
$$

Reconverting to the $a$-oscillator basis gives

$$
\begin{aligned}
|\mathcal{N}\rangle & \approx\left(1-\frac{1}{2} S^{\dagger} S s^{\dagger}\right)|0\rangle_{s} \\
& \approx\left(1-\frac{1}{2} a^{\dagger} S a^{\dagger}\right)\left(1+\frac{1}{2} a^{\dagger} S a^{\dagger}\right)|0\rangle_{a}+\mathcal{O}\left(S^{2}\right) \\
& =|0\rangle_{a}+\mathcal{O}\left(S^{2}\right) .
\end{aligned}
$$

This shows that at high mass levels the field $\mathcal{S}$ dominating the lowest-order solution is cancelled. This is consistent with the expected structure of the nonperturbative vacuum $\mathcal{N}$, with low-mass levels taking values near the squeezed field $\mathcal{S}$ and high-mass levels taking ones close to the usual vacuum.

The contributions to excitation number two arising from the last star-product term in Eq. (83) consist of $P_{2}$ projections on each index of $\mathcal{N}_{20}^{\lambda_{1} \lambda_{2}(1)}$. Similarly, the preceding term involves $P_{1}$ projections. Moreover, the contributions of unmixed projections in the term involving $-s^{\dagger}$ cancel, so the result (84) in fact holds exactly at order $S$ for all mass-level contributions involving unmixed projections. Also, at order $S$ the mixed projections are absent from the final two starproduct terms. However, contributions from the $-s^{\dagger}$ term do arise for the mixed projections. Schematically, one finds in this case that the result $(84)$ for $\mathcal{N}_{20}^{(1)}$ becomes replaced by a structure of the general form $-S E^{-2} /\left(2 E^{-2}-1\right)$. For the subleading mass level this produces a contribution $-S$, thereby changing the sign of the term proportional to $S$ in the lowest-order approximation. Since the true state acquires a combination of contributions from mixed and unmixed projections, one again can anticipate an effect intermediate between $\mathcal{S}$ and the usual vacuum at the lowest mass levels. We see that the nonperturbative vacuum $\mathcal{N}$ can be regarded as a twisted squeezed state, constructed by the action of operators on the squeezed field $\mathcal{S}$ and with net field values dropping more rapidly with excitation number than an exponential at this order of approximation.

The order- $S$ solution can now be used to advance to order $S^{2}$. A nonzero contribution to $\mathcal{N}_{40}^{(2)}$ arises at this level, along with corrections to the lower-order results. This pattern continues at higher orders, with the first contribution to $\mathcal{N}_{2 N, 0}$ being at order $S^{N}$. It would be interesting to determine whether a mechanism analogous to that described above causes higher-order expectation values also to approach zero faster than an exponential as the excitation level increases.

A complete treatment at order $S^{2}$ requires developing a method to handle divergent traces that appear in some terms. For example, a contribution arises to the vacuum-energy shift $\Delta$ in Eq. (74) that is proportional to $\operatorname{Tr}\left(S^{2} E^{-2}\right)$ and that appears to have a linear divergence. It is possible that combining this with the ghost contribution would yield a finite result, but in general it may be necessary to regulate such terms. Zeta-function regularization may be most appropriate, since it is known to avoid an associativity anomaly in the vertex in related calculations [24].

In the ghost sector, an analogous construction for the nonperturbative vacuum $\mathcal{N}$ can be performed. It is straightforward to extend the formal analysis to a solution in powers of $\widetilde{S}$ and to extract results at order $\widetilde{S}$. The order $\widetilde{S}^{0}$ equation has the same form as Eq. (80), so the complete lowest-order solution can be taken as $\mathcal{N}_{00}^{(0)}=|-\rangle_{s, t, u}$ with all other components zero. The equivalent of Eq. (83) for the ghost sector is

$$
\begin{aligned}
& {\left[\left(t^{\dagger} E\left\{\widetilde{S}, E^{-2}\right\} E^{-1} u^{\dagger}\right) \mathcal{N}_{00}^{(0)}+\left[\left(t^{\dagger} E^{-2}\right)_{\mu_{1}} u_{\nu_{1}}^{\dagger}\right.\right.} \\
& \left.\left.\quad+t_{\mu_{1}}^{\dagger}\left(\left(u^{\dagger} E^{-2}\right)_{\nu_{1}}\right)\right] \mathcal{N}_{02}^{\mu_{1} \nu_{1}(1)}+\text { higher-level terms }\right]|+\rangle_{t, u} \\
& \quad+\left|\mathcal{N}^{(1)}\right\rangle \star\left|\mathcal{N}^{(0)}\right\rangle+\left|\mathcal{N}^{(0)}\right\rangle \star\left|\mathcal{N}^{(1)}\right\rangle=0 .
\end{aligned}
$$

Contributions from excitation number greater than 2 and from $\mathcal{N}_{00}^{(1)}$ again can be neglected at this order.

The analogue of Eq. (84) in the limit of high mass level is

$$
\mathcal{N}_{02}^{\mu_{1} \nu_{1}(1)} \approx-\left(E \widetilde{S} E^{-1}\right)^{\mu_{1} \nu_{1}} .
$$

Evidently, the contributions from ghost fields at excitation number 2 become of order $\widetilde{S}^{2}$ in this limit. At low mass levels, an intermediate effect between cancellation of the term linear in $\widetilde{S}$ and a reversal of the sign can again be expected.

\section{B. Properties}

Since the form of our solution is Lorentz invariant by construction, we expect $\mathcal{N}$ to correspond to the Lorentzinvariant nonperturbative vacuum of Refs. [2-4]. The results in the previous subsection can be used to compare the solution for $\mathcal{N}$ with numerical values for nonperturbative solutions obtained in the level-truncation scheme.

In Table I we list numerical approximations for some component bosonic-sector scalar fields in the Lorentinvariant nonperturbative vacuum obtained [8] at level trun- 
cation $(10,20)$, together with the range in which the $\mathcal{O}(\mathcal{S})$ solution for $\mathcal{N}$ indicates the values should lie. The discussion in the previous subsection implies that the true values are expected to lie near the maximum of this range for low-lying states but to fall to the minimum of the range as the level number increases. These results support the identification of $\mathcal{N}$ with the level-truncated Lorentz-invariant nonperturbative vacuum.

In the ghost sector, the numerical values for the ghostoscillator fields follow a pattern similar to that in the bosonic sector. However, a numerical approximation for $\widetilde{S}$ is more involved than for $S$ because approximating $\widetilde{T}_{+}$by Taylor expansion of the square root in Eq. (43) fails for the largest two eigenvalues of $\tilde{X}$, which lie outside the radius of convergence. It would be interesting to find a means of approximating $\widetilde{S}$ with sufficient accuracy to make possible comparisons with truncation-scheme calculations.

At order $\widetilde{S}^{2}$, the level of technical complication increases and the corresponding analysis lies beyond the scope of this work. We conjecture that the cancellation mechanism occurring at linear order in the limit of high mass level generalizes at higher order. This is consistent with the pattern emerging from numerical approximations using the truncation scheme.

It is of interest to compare the vacuum energy $E_{\mathcal{N}}$ of $\mathcal{N}$ to the D25-brane mass $M_{25}$. In principle, it suffices to evaluate the action $I(\Psi)$ for the on-shell string field $\Psi=\mathcal{N}$ and use $E_{\mathcal{N}}=I(\mathcal{N})$. In the present context, the evaluation can be performed directly at order $S, \widetilde{S}$ :

$$
\begin{aligned}
E_{\mathcal{N}}=I(\mathcal{N}) & =\frac{1}{2} \int \mathcal{N} \star Q \mathcal{N}+\frac{1}{3} \int \mathcal{N} \star \mathcal{N} \star \mathcal{N} \\
& =-\frac{1}{6} \int \mathcal{N} \star \mathcal{N} \star \mathcal{N} \\
& \approx-\frac{1}{6} \int \mathcal{S} \star \mathcal{S} \star \mathcal{S} \\
& =-\frac{1}{6} 123, s, t, u\left\langle-\mid V_{3}\right\rangle_{s, t, u} \\
& \approx-\frac{1}{6}+\mathcal{O}\left(S^{2}, \widetilde{S}^{2}\right) \\
& \simeq-0.17
\end{aligned}
$$

This derivation takes advantage of the vanishing of the diagonal elements of the 3-vertex in the $s, t, u$, basis, proved in Sec. IV. The result is to be compared with the expected value $E_{\mathcal{N}}=-M_{25}=-2 / \pi^{2} \approx-0.20$. Approximating the field $\mathcal{N}$ by $\mathcal{S}$ thus gives about $85 \%$ of the D-brane mass.

The string action can also provide insight into the nature of excitations about the squeezed field $\mathcal{S}$. Consider first a string field $\Psi$ expanded about an arbitrary background $\Psi_{B}: \Psi=\Psi_{B}+\Delta$. The action for $\Psi$ can be written [25]

$$
\begin{gathered}
I(\Psi)=\frac{1}{2 \alpha^{\prime}} \int \Psi_{B} \star Q \Psi_{B}+\frac{g}{3} \int \Psi_{B} \star \Psi_{B} \star \Psi_{B} \\
+\frac{1}{2 \alpha^{\prime}} \int \Delta * Q_{B} \Delta+\frac{g}{3} \int \Delta \star \Delta \star \Delta .
\end{gathered}
$$

TABLE I. Vacuum expectation values for bosonic-sector scalar states in the nonperturbative vacuum. For each state, the expectation value $\langle\psi\rangle$ evaluated in the level-truncation scheme and the range allowed by the order- $S$ calculation in the text are presented. The correct value is predicted to be near the maximum of this range for low-lying states but rapidly to approach the minimum as the level number increases, in agreement with the results from the level-truncation scheme.

\begin{tabular}{ccc}
\hline \hline State & $\langle\psi\rangle$ & Expected range \\
\hline$|0\rangle$ & 1.09259 & 1 \\
$a_{1}^{\dagger} \cdot a^{\dagger}|0\rangle$ & 0.05723 & 0 to 0.069 \\
$a_{1}^{\dagger} \cdot a^{\dagger}|0\rangle$ & -0.01988 & -0.053 to 0 \\
$a_{1}^{\dagger} \cdot a^{\dagger}|0\rangle$ & -0.01018 & -0.023 to 0 \\
$a_{2}^{\dagger} \cdot a^{\dagger}|0\rangle$ & 0.00783 & 0 to 0.032 \\
$a^{\dagger} \cdot a_{2} \cdot a^{\dagger}|0\rangle$ & 0.00823 & 0 to 0.031 \\
$a^{\dagger} \cdot a_{3}^{\dagger}|0\rangle$ & 0.00429 & 0 to 0.017 \\
\hline \hline
\end{tabular}

Here, the action of the background Becchi-Rouet-StoraTyutin (BRST) operator $Q_{B}$ on an arbitrary string field $\Phi$ of ghost number $g(\Phi)$ is given by

$$
Q_{B} \Phi=Q \Phi+g \alpha^{\prime}\left[\Psi_{B} \star \Phi-(-1)^{g(\Phi)} \Phi \star \Psi_{B}\right] .
$$

The background operator $Q_{B}$ is nilpotent and distributive across the star product: $Q_{B}\left(\Phi_{1} \star \Phi_{2}\right)=Q_{B} \Phi_{1} \star \Phi_{2}+$ $(-1)^{g\left(\Phi_{1}\right)} \Phi_{1} \star Q_{B} \Phi_{2}$. It also satisfies $\int Q_{B} \Phi_{1} \star \Phi_{2}=$ $(-1)^{g\left(\Phi_{1}\right)+1} \int \Phi_{1} \star Q_{B} \Phi_{2}$. The action $I(\Psi)$ is invariant under

$$
\delta \Delta=\frac{1}{\sqrt{2 \alpha^{\prime}}} Q_{B} \Lambda+g \sqrt{\frac{\alpha^{\prime}}{2}}(\Delta \star \Lambda-\Lambda \star \Lambda),
$$

where $\Lambda$ is a string gauge field.

In the special case that $\Psi_{B}=\mathcal{S}$, the fluctuations $\Delta$ represent oscillations about the squeezed field $\mathcal{S}$ and the background BRST operator $Q_{B}=Q_{S}$ determines the corresponding spectrum. We conjecture that this operator has no tachyonic modes. This would provide further support for the idea that the squeezed field $\mathcal{S}$ provides a useful starting point for investigating the nonperturbative structure of the open bosonic string, despite not being a solution of the string equations of motion.

As partial support for the conjecture, we verify it at order $S$ for low-lying states in the bosonic sector. Consider first the state $|0\rangle_{s}$. The $\mathcal{S}$-background BRST operator $Q_{s}$ acting on this state gives

$$
\begin{aligned}
b_{0} Q_{S}|0\rangle_{s} & \approx\left(s^{\dagger} E^{-2} s-1\right)|0\rangle_{s}+2|0\rangle_{s} \star|0\rangle_{s} \\
& \left.\approx-|0\rangle_{s}+2|0\rangle_{s} \star 0\right\rangle_{s}=+|0\rangle_{s} .
\end{aligned}
$$


This approximation suffices to show that, instead of a tachyonic mass as in the vacuum $|0\rangle_{a}$, the state $|0\rangle_{s}$ acquires a conventional mass.

Similar reasoning supports the conjecture for the first excited states $s_{n}^{\dagger}|0\rangle_{s}$ :

$$
\begin{aligned}
b_{0} Q_{S} s_{n}^{\dagger}|0\rangle_{s} \approx & \left(s^{\dagger} E^{-2} s-1\right) s_{n}^{\dagger}|0\rangle_{s} \\
& +|0\rangle_{s} \star s_{n}^{\dagger}|0\rangle_{s}+s_{n}^{\dagger}|0\rangle_{s} \star|0\rangle_{s} \\
= & (n-1) s_{n}^{\dagger}|0\rangle_{s}+P_{1} s_{n}^{\dagger}|0\rangle_{s} \star|0\rangle_{s} \\
& +|0\rangle_{s} \star P_{2} s_{n}^{\dagger}|0\rangle_{s} \\
= & n s_{n}^{\dagger}|0\rangle_{s} .
\end{aligned}
$$

Since $n>0$, this calculation shows that the analogue of the states $a_{n}^{\dagger}|0\rangle_{a}$ that contain massless modes is here a set of purely massive states $s_{n}^{\dagger}|0\rangle_{s}$.

It is believed that the endpoint of tachyon condensation on the D25-brane has no physical open-string excitations [5]. The corresponding conjecture in the present context is that no normalizable states can be constructed on $\mathcal{N}$. Equivalently, setting $\Psi_{B}=\mathcal{N}$ in the background equations above, one expects the background BRST operator $Q_{B}=Q_{\mathcal{N}}$ to have no normalizable physical fluctuations $\Delta$, so the coefficients of all physical kinetic terms for $\Delta$ should vanish. An alternative would be to show there are no zeros in the euclidean propagators for physical fields in the nonperturbative vacuum [2]. Although our construction of $\mathcal{N}$ sets the momenta to zero, it may be possible to generalize it to incorporate euclidean propagators for $\Delta$ (cf. remarks in the next section). In any event, it would be interesting to have an explicit proof of this conjecture in the context of string field theory.

We note in passing that states on which the action of creation and annihilation operators produces only unnormalizable results can readily be found. For example, in the context of a one-dimensional bosonic oscillator the state $|\psi\rangle$ $=\Sigma_{n}\left[\left(a^{\dagger}\right)^{n} / n \sqrt{n !}\right]|0\rangle$ has finite norm $\Sigma_{n} n^{-2}=\pi^{2} / 6$, but the norms of both $a^{\dagger}|\psi\rangle$ and $a|\psi\rangle$ behave as $\Sigma_{n} n^{-1}$ and so diverge. A multidimensional version of this is the state $\Pi_{m} \Sigma_{n}\left[\left(a_{m}^{\dagger}\right)^{n} / n \sqrt{n !}\right]|0\rangle$, which itself is normalizable but produces unnormalizable states when acted on by any of the oscillator creation and annihilation operators $a_{m}^{\dagger}, a_{m}$. We anticipate that the nonperturbative vacuum $\mathcal{N}$ has similar features.

A related issue is the fate of the low-energy $\mathrm{U}(1)$ or $\mathrm{U}(N)$ particle symmetry of the perturbative massless vectors in the nonperturbative vacuum $\mathcal{N}$. In string field theory, the nonAbelian gauge symmetry is modified compared to the usual transformation law. It has been shown that the nonperturbative vacuum $\mathcal{N}$ maintains the string $\mathrm{U}(N)$ symmetry contained in Eq. (91) in the sense that nonzero scalar expectation values remain invariant under a string $\mathrm{U}(N)$ transformation to all truncation orders [3]. However, this low-energy string symmetry is distinct from the usual $\mathrm{U}(N)$ particle gauge symmetry, with the two being related through a set of nonlinear field redefinitions [26]. The effect of this on the generation of a mass term for the $\mathrm{U}(1)$ field has recently been studied in the context of the level-truncation scheme [27]. Confinement via the condensation of magnetically charged tachyons has been suggested [28], the possibility of a critical value for the $\mathrm{U}(1)$ field above which no solutions exist has been investigated [29], and it has also been proposed that as the tachyon condenses the noncommutative gauge symmetry is fully unbroken and becomes a linearly realized $U(\infty)$ forbidding propagation of open-string modes [30]. It would be interesting to investigate the $\mathrm{U}(N)$ particle symmetry in the context of the solution $\mathcal{N}$ found here. The nonzero mass for vector excitations about the squeezed field $\mathcal{S}$, demonstrated by Eq. (93), suggests this particle symmetry is destroyed in the nonperturbative vacuum.

\section{DISCUSSION}

Using analytical methods, we have constructed a nonperturbative vacuum $\mathcal{N}$ satisfying the full equations of motion (1) of string field theory. It appears to coincide with the Lorentz-invariant nonperturbative vacuum found using the level-truncation scheme and resulting from tachyon condensation on a D25-brane.

In the original $(a, b, c)$-oscillator basis and in the SiegelFeynman gauge, the form of $\mathcal{N}$ is given as

$$
|\mathcal{N}\rangle=F\left(S, a^{\dagger}\right) \widetilde{F}\left(\widetilde{S}, b^{\dagger}, c^{\dagger}\right)|\mathcal{S}\rangle .
$$

Here, the squeezed field $|\mathcal{S}\rangle$ satisfying Eq. (2) is explicitly given in closed form in Eq. (44). Closed-form expressions for the matrices $S=C T_{-}$and $\widetilde{S}=C \widetilde{T}_{+}$in terms of the 3-vertex functions $V^{11}$ and $\widetilde{V}^{11}$ in the momentum representation are determined by Eqs. (39) and (43). The product $F \widetilde{F}$ $\approx 1+\mathcal{O}(S, \widetilde{S})$ is constructed recursively in powers of $S$ and $\widetilde{S}$ in Sec. V.

The string field $\mathcal{N}$ can be regarded as a twisted squeezed state. The construction (94) provides $\mathcal{N}$ in the form of a series of creation operators acting on a squeezed field $\mathcal{S}$. We suspect a closed form exists for this twist series. The rapid decay of the expectation values with level number appears to be a crucial feature of $\mathcal{N}$. The occurrence of the operator $L_{0}$ in the equations of motion and its growth with level number makes it a natural candidate for controlling this rapid decay and hence the twist series, and we therefore conjecture that the closed form for $\mathcal{N}$ is a twisted squeezed state with twist explicitly determined by $L_{0}$. In any case, it would be of interest to develop systematic methods for handling the twist series. Note also that the squeezed field $\mathcal{S}$ itself may merit further study in its own right. Although $\mathcal{S}$ does not solve the equations of motion, it may be a better starting point than the usual perturbative vacuum for approximation schemes studying the properties of $\mathcal{N}$.

An interesting issue is whether solutions exist to Eq. (79) other than $\mathcal{N}$. This would imply that at least some terms with $m \neq 0$ in Eq. (79) are nonzero. Since the star product between states with excitation numbers $m_{1}$ and $m_{2}$ generally yields terms up to excitation number $\left(m_{1}+m_{2}\right)$, it seems likely that nonzero terms persist for arbitrarily high excitation number. Any other solution to Eq. (79) is therefore unlikely to be 
simple within this framework even at low order. Also, satisfying the criterion of normalizability may be more difficult for any such solution because Eq. (79) suggests it must behave roughly as a functional linear in $E_{n n}^{-2}=n$, and hence linear in the mass level.

It may also be possible to obtain other solutions of interest by modifying the construction of $\mathcal{N}$ in a different way. In Sec. V, attention was primarily restricted to states of even excitation number. However, more general possibilities exist. For a bosonic tensor $f_{m n}^{\lambda_{1} \cdots \lambda_{m} \mu_{1} \cdots \mu_{n}}$, we can separate the indices according to left and right sectors so that $f_{m n}$ is annihilated by contracting with $\left(P_{1}\right)_{\mu_{i}}^{\mu_{i}^{\prime}}$ and $\left(P_{2}\right)_{\lambda_{i}}^{\lambda_{i}^{\prime}}$. The action of $L_{0}$ takes $f_{m n}$ to a combination of terms having indices of the type $(m-2, n), \quad(m-1, n-1), \quad(m, n-2), \quad(m, n)$, $(m+1, n-1), \quad(m-1, n+1), \quad(m+2, n), \quad(m+1, n+1)$, $(m, n+2)$. These can be regarded as a field of black squares on a chess board: $(n+m)$ modulo 2 is conserved. Since the star product combines an $(m, k)$ tensor with a $(k, n)$ tensor to yield an $(m, n)$ tensor, it also preserves the sum of the indices modulo 2. The possibility therefore exists of obtaining solutions $f_{m n}$ to the equations of motion restricted to even $(m+n)$ other than the nonperturbative vacuum $\mathcal{N}$.

Yet another possibility is to consider solutions mixing the colors on the chessboard. Unlike solutions restricted to even $(m+n)$, which in general may or may not violate Lorentz symmetry, solutions with odd $(m+n)$ would have an odd number of oscillators and hence would necessarily violate Lorentz symmetry. In string field theory, the occurrence of static interaction terms quadratic in tensors and linear in scalars suggests the possibility of spontaneous breaking of Lorentz symmetry [31], which would be accompanied by $C P T$ violation when fields with odd level number are involved [32]. Indeed, a family of Lorentz-violating and CPTpreserving solutions in the open bosonic string has been uncovered using the level-truncation scheme in the open bosonic string [4]. It would be interesting to construct analytically the states corresponding to these solutions.

In general, the topic of Lorentz-violating solutions appears to be a potentially revealing subject for future study. Relatively little is known about the nature of these solutions, including whether they are stable. Since they are spacetime independent, it appears unlikely that they could correspond to any of the lump solutions discussed in the literature to date. The ubiquitous nature of the cubic scalar-tensor-tensor couplings suggests that Lorentz-violating solutions are generic in string field theories with tachyons. In fact, the existence of scalar-tensor-tensor couplings is not restricted to string field theory, since they also arise in off-shell calculations of the action using renormalization-group methods in the string sigma model [26].

Since the physical world is believed to include a tachyon in its spectrum at the electroweak scale (the Higgs field), it is necessary for a realistic string theory to have at least one tachyonic mode. If Lorentz-violating solutions are indeed generic in theories with tachyons, it is natural to speculate that the four-dimensional Lorentz and CPT symmetries might also be spontaneously broken. The resulting low-energy effects can be systematically studied [33]. The exquisite sensi- tivity of modern experiments to such effects [34-42] means that even effects suppressed by the Planck scale could be detected.

Another interesting class of objects in string field theory is the set of lump solutions. Using the level-truncation scheme, it has been shown that bosonic D $p$-branes can be interpreted as unstable lump configurations of open-string tachyons on a D25-brane [43], at least for large $p$. Worldsheet-boundary renormalization-group methods can be used to show that the mass of a tachyonic lump on a $\mathrm{D} p$-brane corresponds to that of a $\mathrm{D}(p-1)$-brane [44]. This has also been verified numerically in a modified leveltruncation scheme incorporating derivative couplings [45]. Some of these solutions are related to results in noncommutative field theory [46]. Various other calculations support these ideas $[47,48]$.

It is plausible that a variant of our methodology could be used to construct the lump solutions analytically. In particular, the construction of $\mathcal{N}$ depends only on the formal structure of the vertex functions in terms of the matrices $C$ and $U$ with properties (12). These matrices emerge from the conversion between the $a_{0}$ oscillators and the momentum representation in Sec. II A. They originate in a set of matrices $C$ and $U^{\prime}$ with identical formal properties (9). It therefore follows that any spacetime-independent solution obtained in the momentum representation has an inequivalent partner solution in the $a_{0}$ representation. Moreover, the conversion from oscillator to momentum basis can be performed in any number $p$ of spacetime dimensions desired. The construction implemented for $\mathcal{N}$ therefore suggests the existence of string fields that are twisted squeezed states in $(26-p)$ dimensions and are independent of the remaining $p$ dimensions. We conjecture that these solutions include the $\mathrm{D} p$-branes, along with instanton-type solutions.

Another topic of interest is the role and fate of the closedstring modes in $\mathcal{N}$. The Zwiebach string field theory of the closed bosonic string is nonpolynomial [49]. Consistent implementation of the level-truncation scheme therefore presents some difficulties. At the level of the cubic interaction, a nonperturbative solution appears to exist in which the closed-string tachyon and the graviton have no physical poles [50]. We anticipate that a closed-string version of the squeezed field $\mathcal{S}$ can be constructed for this solution. If so, its idempotency may make possible analytical study of the nonpolynomial action in the squeezed basis. It has also been shown that macroscopic closed strings can be regarded as solitons in the nonperturbative vacuum of the open bosonic string [51]. It would be interesting to study solutions of this type in the squeezed-state basis.

The methodology in this paper could also be applied to study the nonperturbative vacua of other string field theories with tachyons, including those for the non-GSO-projected superstring [9] and the $p$-adic string [52]. For the latter, it has explicitly been proved that the extremum of the tachyon potential with a D25-brane is the vacuum without one and that lump solutions are D-branes of lower dimensions [53]. In light of the possibility of obtaining exact results, it would be interesting to investigate our construction for $\mathcal{N}$ in this context. 


\section{ACKNOWLEDGMENTS}

This work is supported in part by the United States Department of Energy under Grant No. DE-FG02-91ER40661 and by the Portuguese Fundação para a Ciência e a Tecnologia.

\section{APPENDIX: A USEFUL IDENTITY}

This Appendix explicitly proves the identity (49) for multidimensional bosonic oscillators under the conditions (50). A similar method can be adopted to prove the result (66) for multidimensional ghost oscillators subject to the constraints (67).

The strategy we adopt is to seek a scalar function $\eta(t)$ of a parameter $t$ and matrix functions $\alpha_{m n}(t), \beta_{m n}(t), \gamma_{m n}(t)$ of the same parameter $t$ such that the equation

$$
\begin{aligned}
\exp [ & \left.t\left(a_{m}^{\dagger} A_{m n} a_{n}^{\dagger}+a_{m}^{\dagger} C_{m n} a_{n}+a_{m} B_{m n} a_{n}\right)\right] \\
= & \exp (\eta) \exp \left(a_{m}^{\dagger} \alpha_{m n} a_{n}^{\dagger}\right) \\
& \times \exp \left(a_{m}^{\dagger} \gamma_{m n} a_{n}\right) \exp \left(a_{m} \beta_{m n} a_{n}\right)
\end{aligned}
$$

holds. Here, the matrices $A, B$, and $C$ are assumed independent of $t$. The idea is to take the derivative with respect to $t$ on both sides of the equation, commute to the left all factors that appear, and equate the corresponding terms on both sides. This yields differential equations for the scalar and matrix functions of $t$. Solving these yields the desired identity by setting $t=1$.

Adopting this procedure while keeping careful track of the index positions yields the following set of matrix differential equations:

$$
\begin{aligned}
& A=\dot{\alpha}-2 \dot{\gamma} \alpha+4 \alpha \exp \left(-\gamma^{T}\right) \dot{\beta} \exp (-\gamma) \alpha, \\
& B=\exp \left(-\gamma^{T}\right) \dot{\beta} \exp (-\gamma), \\
& C=\dot{\gamma}-4 \alpha \exp \left(-\gamma^{T}\right) \dot{\beta} \exp (-\gamma), \\
& 0=\dot{\eta}-2 \operatorname{Tr}\left[\exp \left(-\gamma^{T}\right) \dot{\beta} \exp (-\gamma) \alpha\right],
\end{aligned}
$$

where $T$ denotes the transpose. For simplicity in the above and in what follows, we assume

$$
\begin{aligned}
& \alpha^{T}=\alpha, \quad \alpha C^{T}=C \alpha, \\
& \beta^{T}=\beta, \quad \beta C=C^{T} \beta
\end{aligned}
$$

as a partial ansatz that is to be verified after the solution is constructed. The equations (A2) can be rearranged to give

$$
\begin{aligned}
& \dot{\alpha}=A+2 C \alpha+4 \alpha B \alpha, \\
& \dot{\beta}=e^{\gamma^{T}} B e^{\gamma}, \\
& \dot{\gamma}=C+4 \alpha B, \\
& \dot{\eta}=2 \operatorname{Tr}(B \alpha) .
\end{aligned}
$$

As initial conditions for these equations, we take $\alpha(0)$ $=\beta(0)=\gamma(0)=\eta(0)=0$.

Taking advantage of the given conditions (50), Eq. (A4) becomes

$$
\dot{\alpha}=\left(\alpha+\frac{1}{4} C B^{-1}\right) 4 B\left(\alpha+\frac{1}{4} C B^{-1}\right) .
$$

Its solution is $\alpha+\frac{1}{4} C B^{-1}=(-4 B t+Y)^{-1}$, where the initial condition fixes $Y=4 B C^{-1}$. This gives

$$
\alpha(t)=(1-C t)^{-1} A t .
$$

Substituting this into Eq. (A6) gives $\dot{\gamma}=-\left(t-C^{-1}\right)^{-1}$, which has solution

$$
\gamma(t)=-\ln (1-C t)
$$

This in turn can be substituted into Eq. (A5), leading to $\dot{\beta}$ $=B(1-C t)^{-2}$. The solution is

$$
\beta(t)=B t(1-C t)^{-1} .
$$

This leaves Eq. (A7), which becomes

$$
\dot{\eta}=\frac{1}{2} \operatorname{Tr}\left[\left((1-C t)^{-1}-1\right) C\right]
$$

with solution

$$
\eta(t)=\frac{1}{2} \operatorname{Tr}[-\ln (1-C t)-C t] .
$$

We can now verify the ansatz (A3). Finally, setting $t=1$ yields the desired identity (49).
[1] E. Witten, Nucl. Phys. B268, 253 (1986).

[2] V. A. Kostelecký and S. Samuel, Phys. Lett. B 207, 169 (1988); Nucl. Phys. B336, 263 (1990).

[3] V. A. Kostelecký and S. Samuel, Phys. Rev. Lett. 64, 2238 (1990).

[4] V. A. Kostelecký and R. Potting, Phys. Lett. B 381, 89 (1996).

[5] A. Sen, J. High Energy Phys. 08, 012 (1998); 12, 027 (1999).

[6] A. Sen and B. Zwiebach, J. High Energy Phys. 03, 002 (2000).

[7] W. Taylor, Nucl. Phys. B585, 171 (2000).

[8] N. Moeller and W. Taylor, Nucl. Phys. B583, 105 (2000).

[9] N. Berkovits, J. High Energy Phys. 04, 022 (2000).
[10] N. Berkovits, A. Sen, and B. Zwiebach, Nucl. Phys. B587, 147 (2000).

[11] P. De Smet and J. Raeymaekers, J. High Energy Phys. 05, 051 (2000).

[12] J. David, J. High Energy Phys. 10, 004 (2000).

[13] V. A. Kostelecký (unpublished).

[14] See, for example, A. Perelomov, Generalized Coherent States and Their Applications (Springer-Verlag, New York, 1986); M. M. Nieto, in Frontiers of Nonequilibrium Statistical Physics, edited by G. T. Moore and M. O. Scully (Plenum, New York, 1986). 
[15] Equations of the Riccati form often appear in the construction of coherent and squeezed states for an operator algebra. In many cases, the existence of the algebra makes possible their solution despite their superficial intractability. See, for example, the analytical solution of a system of 14 simultaneous coupled nonlinear differential equations arising for the superPoincaré group, V. A. Kostelecký and D. R. Truax, J. Math. Phys. 28, 2480 (1987). In string field theory, the Riccati form of the equations of motion and the presence of the string gauge symmetry are also suggestive of a coherent- or squeezed-state solution.

[16] D. J. Gross and A. Jevicki, Nucl. Phys. B283, 1 (1987); B287, 225 (1987).

[17] E. Cremmer, A. Schwimmer, and C. Thorn, Phys. Lett. B 179, 57 (1986).

[18] S. Samuel, Phys. Lett. B 181, 255 (1986).

[19] The application of these equations in later sections is in the context of the Feynman-Siegel gauge [21]. This means that the usual zero modes of the ghost algebra can be disregarded here.

[20] The vacua $| \pm\rangle$ are defined following Ref. [16]. For a single oscillator, $| \pm\rangle$ are the ghost-number $\pm \frac{1}{2}$ vacua satisfying $|+\rangle=c_{0}|-\rangle,|-\rangle=b_{0}|+\rangle$.

[21] W. Siegel, Phys. Lett. B 142, 276 (1984); 151, 391 (1985); 151, 396 (1985).

[22] L. Rastelli and B. Zwiebach, hep-th/0006240.

[23] J. Bordes, H.-M. Chan, L. Nellen, and S. T. Tsou, Nucl. Phys. B351, 441 (1991).

[24] R. Potting and C. Taylor, Nucl. Phys. B316, 59 (1989).

[25] V. A. Kostelecký and S. Samuel, in Superstrings and Particle Theory, edited by L. Clavelli and B. Harms (World Scientific, Singapore, 1990).

[26] V. A. Kostelecký, R. Potting, and M. Perry, Phys. Rev. Lett. 84, 4541 (2000).

[27] W. Taylor, J. High Energy Phys. 08, 038 (2000).

[28] O. Bergman, K. Hori, and P. Yi, Nucl. Phys. B580, 289 (2000).

[29] A. Sen and B. Zwiebach, J. High Energy Phys. 10, 009 (2000).

[30] R. Gopakumar, S. Minwalla, and A. Strominger, hep-th/0007226.
[31] V. A. Kostelecký and S. Samuel, Phys. Rev. D 39, 683 (1989).

[32] V. A. Kostelecký and R. Potting, Nucl. Phys. B359, 545 (1991).

[33] V. A. Kostelecký and R. Potting, Phys. Rev. D 51, 3923 (1995); D. Colladay and V. A. Kostelecký, ibid. 55, 6760 (1997); 58, 116002 (1998). The literature discussing specific experiments can be traced from the references in V. A. Kostelecký, ibid. 61, 016002 (2000); R. Bluhm and V. A. Kostelecký, Phys. Rev. Lett. 84, 1381 (2000).

[34] KTeV Collaboration, Y. B. Hsiung et al., Proceedings of the 1999 Chicago Conference on Kaon Physics, Chicago, Illinois, 1999, Report No. FERMILAB-CONF-99-313-E.

[35] OPAL Collaboration, R. Ackerstaff et al., Z. Phys. C 76, 401 (1997); DELPHI Collaboration, M. Feindt et al., Report No. DELPHI 97-98 CONF 80, 1997.

[36] L. R. Hunter et al., in CPT and Lorentz Symmetry, edited by V. A. Kostelecký (World Scientific, Singapore, 1999).

[37] G. Gabrielse et al., Phys. Rev. Lett. 82, 3198 (1999).

[38] R. Mittleman et al., Phys. Rev. Lett. 83, 2116 (1999).

[39] H. G. Dehmelt et al., Phys. Rev. Lett. 83, 4694 (1999).

[40] D. Bear et al., Phys. Rev. Lett. 85, 5038 (2000).

[41] R. Walsworth et al., physics/0007063; D. F. Phillips et al., physics/0008230.

[42] B. Heckel et al., to appear in the proceedings of Orbis Scientiae 1999.

[43] J. Harvey and P. Kraus, J. High Energy Phys. 04, 012 (2000).

[44] J. Harvey, D. Kutasov, and E. Martinec, hep-th/0003101.

[45] N. Moeller, A. Sen, and B. Zwiebach, J. High Energy Phys. 08, 039 (2000).

[46] E. Witten, hep-th/0006071.

[47] J. Majumder and A. Sen, J. High Energy Phys. 06, 010 (2000).

[48] N. Moeller, hep-th/0008101.

[49] B. Zwiebach, Nucl. Phys. B390, 33 (1993).

[50] V. A. Kostelecký and S. Samuel, Phys. Rev. D 42, 1289 (1990).

[51] J. A. Harvey, P. Kraus, F. Larsen, and E. J. Martinec, J. High Energy Phys. 07, 042 (2000).

[52] L. Brekke, P. G. O. Freund, M. Olson, and E. Witten, Nucl. Phys. B302, 365 (1988).

[53] D. Ghoshal and A. Sen, Nucl. Phys. B584, 300 (2000). 